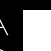
(n)

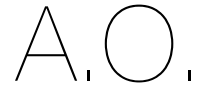

ARTIGO ORIGINAL

${ }^{1}$ Faculdade de Medicina

da Universidade de Lisboa Av. Prof. Egas Moniz, 1649-028 Lisboa, Portugal

2 Escola Superior de Tecnologia da Saúde de Lisboa do Instituto Politécnico de Lisboa, Av. D. João II, Lote 4.69.01, 1990-096 Lisboa, Portugal

${ }^{3}$ H\&TRC, Centro de Investigação em Saúde e Tecnologia,

Av. D. João II, Lote 4.69.01, 1990-096 Lisboa, Portugal

`Endereço para correspondência:

Ana Sofia Teodoro e Sá Esteves Rua Major Cunha, lote 13 , 8670-007 Aljezur, Faro, Portuga asesteves2@gmail.com

Histórico do artigo:

Recebido a 26 de outubro de 2018 Aceite a 15 de setembro de 2019

\section{QUALIDADE DE VIDA EM DOENTES COM EXCESSO PONDERAL}

\author{
QUALITY OF LIFE IN OVERWEIGHT PATIENTS
}

\section{Ana Sofia Esteves ${ }^{1,2} ;$ Ana Catarina Moreira ${ }^{2,3}$}

\section{RESUMO}

INTRODUÇÃo: A obesidade está fortemente associada à morbidade e mortalidade, no entanto é menos claro o seu impacto na qualidade de vida relacionada com a saúde. Devido ao aumento da prevalência de excesso ponderal e suas consequências na saúde e qualidade de vida relacionada com a saúde torna-se importante proceder à sua avaliação.

OBJETIVOS: Avaliar a qualidade de vida relacionada com a saúde antes e após intervenção nutricional para redução ponderal. METODOLOGIA Estudo analítico longitudinal com intervenção nutricional para perda de excesso ponderal/adiposidade, numa amostra de 39 utentes. A qualidade de vida relacionada com a saúde foi avaliada pela aplicação dos questionários SF-36v2 e EQ-5D-3L. RESULTADOS: O peso, a massa gorda e o índice de massa corporal iniciais encontraram-se negativamente associadas a pelo menos uma das dimensões avaliadas. No final da intervenção observou-se uma diminuição de medidas antropométricas e um aumento significativo na qualidade de vida relacionada com a saúde. Verificou-se um aumento do índice EQ-5D-3L com a redução do peso, de índice de massa corporal e de massa gorda.

CONCLUSÕES: A redução ponderal de apenas 4,3\% numa amostra de indivíduos com índice de massa corporal médio classificado em obesidade, embora borderline $\left(30,6 \mathrm{Kg} / \mathrm{m}^{2}\right)$, foi suficiente para se observarem alterações positivas na qualidade de vida relacionada com a saúde.

\section{PALAVRAS-CHAVE}

Excesso de peso, Qualidade de vida relacionada com a saúde, EQ-5D-3L e SF-36

\section{ABSTRACT}

INTRODUCTION: Obesity is strongly associated with morbidity and mortality, but the impact on health-related quality of life is less known. Due to the increasing importance given to health-related quality of life and the higher prevalence of excess weight and its consequences in Health, it is essential to evaluate it.

OBJECTIVE: Evaluate health-related quality of life before and after a nutritional intervention for weight/adiposity reduction. METHODOLOGY: Longitudinal analytical study with nutritional intervention for loss of overweight, in a sample of 39 patients the healthrelated quality of life was assessed by SF-36v2 and EQ-5D-3L questionnaires.

RESULTS: Weight, fat mass and body mass index were negatively associated with at least one of the dimensions evaluated. By the end of intervention the reduction in all anthropometric measurements was follow by significant increase in health-related quality of life. There was an increase in the EQ-5D-3L index with weight reduction, body mass index and fat mass. CONCLUSIONS: The weight reduction of only $4.3 \%$ in a sample of individuals with a moderate body mass index classified as obesity, although borderline $\left(30.6 \mathrm{Kg} / \mathrm{m}^{2}\right)$, was sufficient to observe positive changes in health-related quality of life.

KEYWORDS

Overweight, Health-related quality of life, EQ-5D-3L and SF-36

\section{INTRODUÇÃO}

A obesidade é considerada uma epidemia a nível mundial, e está fortemente associada à morbidade e mortalidade (1). Além do impacto físico, tem um impacto relevante na vertente psicológica e de bem-estar dos indivíduos, podendo afetar a sua qualidade de vida (QV) (2).

No passado, as medições em saúde baseavam-se na presença ou ausência de estados negativos de saúde, limitações funcionais, sintomas de doença e existência de problemas agudos e/ou crónicos (3). Atualmente incluem-se medidas de QV, pelo que têm sido desenvolvidos vários instrumentos que permitem, numa definição global, avaliar a perceção do utente sobre o seu estado de saúde. A QV foi definida pela Organização Mundial da Saúde (OMS) como "a perceção do indivíduo da sua posição na vida no contexto da cultura e sistema de valores nos quais ele vive e em relação aos seus objetivos, expectativas, padrões e preocupações" (2). A qualidade de vlda relacionada com a saúde (QVRS) é um subconjunto dos aspetos de QV relacionados, na existência individual, com o domínio da 
saúde (3-5). São vários os instrumentos de avaliação de QVRS. De entre estes encontra-se o questionário Short Form Health Survey 36 (SF-36) (3), com grande potencial na utilização e o muito utilizado EQ-5D (6). A obesidade parece estar associada a menor QVRS independentemente da existência ou não de outras doenças crónicas (7-9). No entanto são ainda escassos os estudos sobre o impacto da redução ponderal na QVRS.

Devido ao aumento da prevalência de excesso ponderal e suas consequências na saúde, torna-se pertinente avaliar a QVRS e qual o impacto da redução ponderal.

\section{OBJETIVOS}

Foram nossos objetivos, avaliar a QVRS na perda ponderal, bem como avaliar a QVRS em indivíduos com excesso ponderal/adiposidade; e relacionar a recente perda ponderal/adiposidade com a QVRS.

\section{METODOLOGIA}

Tratou-se de um estudo analítico longitudinal, numa população de indivíduos sujeitos a intervenção nutricional para perda de excesso ponderal/adiposidade. A amostra foi constituída por 39 utentes e selecionada por conveniência.

Para ser incluído no estudo o participante tinha de ser adulto e encontrar-se com excesso ponderal e/ou de adiposidade. Foi considerado excesso ponderal/adiposidade a presença de pelo menos um dos seguintes critérios:

1. Perímetro da cintura $(P C)$ aumentado. Considerou-se aumentado quando no género masculino $P C \geq 94 \mathrm{~cm}$ e no género feminino $P C \geq 80 \mathrm{~cm}$ (10). Avaliou-se com recurso a uma fita métrica não extensível Seca ${ }^{\circledR}$ 201, com capacidade até $205 \mathrm{~cm}$ e precisão de $0,1 \mathrm{~cm}$;

2. Percentagem de massa gorda aumentada. Considerou-se aumentada quando $\geq 25 \%$ no género masculino e $\geq 32 \%$ no género feminino. Avaliou-se com recurso a uma balança Tanita ${ }^{\circledR}$ BC-60, com precisão de $100 \mathrm{~g}$;

3. Razão cintura/altura aumentado/a. Considerou-se aumentado quando $\mathrm{RCA}>0,5 \mathrm{~cm}$ (10);

4. Excesso de peso. Considerou-se excesso de peso quando $I M C \geq 25$ $\mathrm{Kg} / \mathrm{m}^{2}$. O IMC foi classificado segundo a OMS (10). Avaliou-se com recurso a uma balança Tanita ${ }^{\circledR}$ BC-60, com precisão de $100 \mathrm{~g}$.

Foram recolhidos dados sociodemográficos (idade e género), diretamente questionados ao utente. Foram ainda avaliados dados de antropometria (altura, peso) e composição corporal (\% massa gorda).Os dados antropométricos e de composição corporal foram avaliados em consulta de acordo com as diretrizes da OMS (10) e DGS (11). Os dados relativos à QVRS foram recolhidos com recurso aos questionários SF-36 v2 (3) e EQ-5D-3L (EuroQol-5 dimensões-3 níveis) e EQ-VAS (EuroQol-escala visual analógica), onde valores mais elevados correspondem a melhor QVRS (6), preenchidos em local reservado e entregues num envelope fechado. A sua aplicação e a avaliação de medidas antropométricas realizou-se em dois momentos: $1 .{ }^{\circ}$ Momento: $1 .{ }^{a}$ consulta de perda ponderal/adiposidade; $\mathrm{e}$ 2. ${ }^{\circ}$ Momento: 8 semanas \pm 2 semanas após o $1 .{ }^{\circ}$ momento.

A análise estatística foi realizada com o software IBM SPSS versão 24.0, utilizou-se um nível de significância $p \leq 0,05$. Na caraterização global da amostra, as variáveis numéricas são resumidas através da média e desvio-padrão (DP) e as variáveis qualitativas através das frequências absolutas e relativas. Para testar a normalidade das variáveis foi utilizado o teste Shapiro-Wilk. Foi realizada uma análise das variáveis através de testes estatísticos paramétricos e não paramétricos, conforme adequado. No caso das variáveis numéricas, a comparação entre os dois momentos foi efetuada através do teste t para duas amostras emparelhadas ou teste Wilcoxon. Para avaliar a alteração das variáveis qualitativas entre dois momentos, recorreu-se ao Teste de Homogeneidade Marginal. Para comparar os valores das variáveis MSF, MSM e dimensões do SF-36, EQ-5D-3L e EQ-VAS entre as diferentes categorias de IMC, utilizou-se o teste Kruskal-Wallis. Com vista a avaliar correlação entre os dados recolhidos utilizou-se os testes à significância de correlação de Pearson e Spearman.

O presente estudo foi aprovado pelo Conselho Científico da Faculdade de Medicina da Universidade de Lisboa e pela Comissão de Ética do Centro Hospitalar Lisboa Norte e da Comissão de Ética do Centro Académico de Medicina de Lisboa. Todos os participantes preencheram o Termo de Consentimento Informado, esclarecido e livre (por escrito e em duplicado).

\section{RESULTADOS}

O tempo decorrido entre as avaliações inicial e final foi de 8 semanas \pm 2 semanas. Verificou-se $20,4 \%$ de dropouts.

\section{Características sociodemográficas e estado nutricional}

Foram incluídos 39 participantes, 82,1\% ( $n=32)$ eram do género feminino. A idade dos participantes variou entre os 19 e 59 anos, apresentando uma média de $39 \pm 11$ anos. A altura média foi $1,65 \pm 0,08 \mathrm{~m}(1,52-1,87)$.

As variáveis referentes ao estado nutricional (refere-se a IMC, RCA, MG e PC) no início e término do estudo apresentam-se em tabela (Tabela 1). Verificou-se uma redução significativa nas variáveis de peso $(3,6$ $\mathrm{Kg} ; 4,3 \%)$, de IMC (1,3 Kg/m²; 4,4\%), de MG (2,3\%), de PC (7,01 cm; $6,8 \%)$ e de RCA $(0,12 \mathrm{~cm} ; 17,7 \%)$.

Verificou-se variação $(p<0,001)$ na classificação nutricional no início e o término do estudo (Tabela 2).

\section{Tabela 1}

Estado nutricional inicial e final

\begin{tabular}{lccc} 
& $\begin{array}{c}\text { INICIAL } \\
\mathrm{n}=39\end{array}$ & $\begin{array}{c}\text { FINAL } \\
\mathrm{n}=39\end{array}$ & $p$ \\
\hline Peso $(\mathrm{Kg})$ & $83,9 \pm 17,9$ & $80,4 \pm 17,5$ & $<\mathbf{0 , 0 0 1 ^ { * }}$ \\
\hline $\begin{array}{l}\text { Índice de Massa Corporal } \\
\left(\mathrm{Kg} / \mathrm{m}^{2}\right)\end{array}$ & $30,6 \pm 4,6$ & $29,3 \pm 4,5$ & $<\mathbf{0 , 0 0 1 ^ { * }}$ \\
\hline Massa gorda $(\%)$ & $37,4 \pm 5,4$ & $35,1 \pm 5,8$ & $<\mathbf{0 , 0 0 1 ^ { * }}$ \\
\hline Perímetro da cintura $(\mathrm{cm})$ & $101,81 \pm 11,35$ & $94,75 \pm 11,38$ & $<\mathbf{0 , 0 0 1 ^ { * }}$ \\
\hline Razão cintura/altura $(\mathrm{cm})$ & $0,61 \pm 0,07$ & $\mathbf{0 , 5 8 \pm 0 , 0 7}$ & $<\mathbf{0 0 0 1}^{*}$ \\
\hline
\end{tabular}

Teste: Teste $t^{\star}$ Diferença entre sexos

\section{Tabela 2}

Valores do Índice de Massa Corporal, massa gorda (\%), perímetro da cintura e razão cintura/altura

\begin{tabular}{|c|c|c|c|c|c|}
\hline & & \multicolumn{2}{|c|}{$\begin{array}{c}\text { INICIAL } \\
\mathrm{n}=39\end{array}$} & \multicolumn{2}{|c|}{$\begin{array}{l}\text { FINAL } \\
\mathrm{n}=39\end{array}$} \\
\hline & & $\mathrm{n}$ & $\%$ & $\mathrm{n}$ & $\%$ \\
\hline \multirow{5}{*}{$\begin{array}{l}\text { Índice de Massa } \\
\text { Corporal }\end{array}$} & Eutrofia & 4 & 10,3 & 6 & $15,4 \%$ \\
\hline & Pré-obesidade & 14 & 35,9 & 18 & $46,2 \%$ \\
\hline & Obesidade I & 14 & 35,9 & 13 & $33,3 \%$ \\
\hline & Obesidade II & 6 & 15,4 & 1 & $2,6 \%$ \\
\hline & Obesidade III & 1 & 2,6 & 1 & $2,6 \%$ \\
\hline \multirow{2}{*}{$\begin{array}{l}\text { Massa gorda } \\
(\%)\end{array}$} & Normal & 2 & 5,1 & 5 & $12,8 \%$ \\
\hline & Em excesso & 37 & 94,9 & 34 & $87,2 \%$ \\
\hline \multirow{3}{*}{$\begin{array}{l}\text { Perímetro da } \\
\text { cintura }(\mathrm{cm})\end{array}$} & Normal & 3 & 7,7 & 5 & $13,2 \%$ \\
\hline & Aumentado & 6 & 15,4 & 7 & $18,4 \%$ \\
\hline & Muito aumentado & 30 & 76,9 & 26 & $68,4 \%$ \\
\hline \multirow{2}{*}{$\begin{array}{l}\text { Razão cintura/ } \\
\text { altura }(\mathrm{cm})\end{array}$} & Normal & 4 & 10,3 & 13 & $33,3 \%$ \\
\hline & Aumentada & 35 & 89,7 & 26 & $66,7 \%$ \\
\hline
\end{tabular}


A maioria dos utentes apresentava no início do estudo MG, PC e RCA em excesso, tendo-se verificado no final do estudo redução no número de utentes classificados na categoria de PC muito aumentado, $M G$ em excesso e RCA, no entanto apenas a última com significado estatístico $(p=0,003)$.

\section{Qualidade de vida relacionada com a saúde}

Do início para o final do estudo, as pontuações do EQ-5D-3L (Tabela 3) aumentaram no EQ-VAS ( $p<0,001)$ e no índice EQ-5D-3L ( $p=0,022)$. Quanto às pontuações do SF-36 (Tabela 3) também foi observado aumento em todas as dimensões, no entanto apenas estatisticamente significativo nas dimensões função física $(p=0,027)$, saúde geral $(p=0,013)$, vitalidade $(p=0,016)$ e MSF $(p=0,015)$.

\section{Influência do estado nutricional na qualidade de vida relacionada com a saúde}

Foi observada (Tabela 4) uma correlação entre o estado nutricional e a QVRS. Observou-se um aumento de diferentes dimensões de QVRS do SF-36 com a redução do IMC, massa gorda, PC e RCA. Não se verificou correlação de nenhuma variável do estado nutricional com o EQ-VAS e com o índice EQ-5D-3L.

A QVRS avaliada pelo SF-36 foi inferior nas categorias mais elevadas de IMC, onde os utentes classificados com excesso ponderal apresentaram pior QVRS nas dimensões função física (96,67 vs. 91,6; $p=0,036)$, dor física $(88,33$ vs. 71,$81 ; \mathrm{p}=0,045)$ e MSF $(59,10$ vs. 57,52; $\mathrm{p}=0,015)$. Nas dimensões de QVRS avaliadas pelo EQ-5D-3L, não houve diferença.
Tabela 3

Pontuações do EQ-VAS, índice EQ-5D-SL e dimensões SF-36

\begin{tabular}{lccccc} 
& \multicolumn{2}{c}{ INICIAL } & \multicolumn{2}{c}{ FINAL } \\
& n=39 & \\
\cline { 2 - 5 } & MÉDIA & DP & MÉDIA & DP & \\
EQ-VAS & 73,7 & 15,9 & 81,4 & 13,0 & $<0,001$ \\
\hline Índice EQ-5D-3L & 0,6 & 0,2 & 0,7 & 0,3 & 0,022 \\
\hline SF-36: Função física & 83,0 & 16,2 & 98,7 & 14,4 & $\mathbf{0 , 0 2 7 ^ { * }}$ \\
\hline $\begin{array}{l}\text { SF-36: Desempenho } \\
\text { físico }\end{array}$ & 83,9 & 17,5 & 86,9 & 13,8 & 0,226 \\
\hline SF-36: Dor física & 69,1 & 19,4 & 74,4 & 15,2 & 0,094 \\
\hline SF-36: Saúde geral & 64,5 & 18,7 & 71,9 & 17,7 & $\mathbf{0 , 0 1 3 ^ { * }}$ \\
\hline SF-36: Vitalidade & 57,3 & 15,8 & 63,6 & 17,4 & $\mathbf{0 , 0 1 6 ^ { * }}$ \\
\hline SF-36: Função social & 75,9 & 20,0 & 78,6 & 18,8 & 0,339 \\
\hline $\begin{array}{l}\text { SF-36: Desempenho } \\
\text { emocional }\end{array}$ & 78,1 & 17,4 & 82,0 & 18,8 & 0,210 \\
\hline SF-36: Saúde mental & 68,1 & 15,5 & 73,0 & 16,0 & 0,067 \\
\hline Medida sumária física & 56,9 & 1,6 & 57,8 & 1,9 & $\mathbf{0 , 0 1 5 ^ { * }}$ \\
\hline Medida sumária mental & 56,9 & 1,6 & 56,0 & 2,3 & 0,057
\end{tabular}

Nota: ${ }^{*} \mathrm{p}<0,05$

DP: Desvio-padrão

EQ-VAS: Escala analógica visual vertical de 0 a 100, sendo 100 o melhor estado de saúde Índice EQ-5D-3L: Sistema descritivo do questionário EQ-5D-3L que varia entre 0 e 1 , sendo o 1 um perfeito estado de saúde

SF-36: Questionário Short Form 36

Teste: Teste t

\section{Tabela 4}

Associação das imagens atual e desejada com o IMC-para-idade

\begin{tabular}{|c|c|c|c|c|c|c|c|c|c|}
\hline & & \multicolumn{4}{|c|}{$\begin{array}{c}\text { INICIAL } \\
\mathrm{n}=39\end{array}$} & \multicolumn{4}{|c|}{$\begin{array}{c}\text { FINAL } \\
\mathrm{n}=39\end{array}$} \\
\hline & & $\begin{array}{l}\mathrm{IMC} \\
\left(\mathrm{Kg} / \mathrm{m}^{2}\right)\end{array}$ & $\begin{array}{l}\text { MASSA GORDA } \\
(\%)\end{array}$ & $\begin{array}{l}\text { PC } \\
(\mathrm{cm})\end{array}$ & $\begin{array}{l}\mathrm{RCA} \\
(\mathrm{cm})\end{array}$ & $\underset{\left(\mathrm{Kg} / \mathrm{m}^{2}\right)}{\mathrm{IMC}}$ & $\begin{array}{l}\text { MASSA GORDA } \\
(\%)\end{array}$ & $\begin{array}{l}\mathrm{PC} \\
(\mathrm{cm})\end{array}$ & $\begin{array}{l}\mathrm{RCA} \\
(\mathrm{cm})\end{array}$ \\
\hline \multirow{2}{*}{ EQ-VAS } & $r$ & $-0,106$ & $-0,127$ & $-0,055$ & 0,052 & $-0,151$ & $-0,177$ & $-0,107$ & 0,055 \\
\hline & $\mathrm{p}$ & 0,473 & 0,389 & 0,714 & 0,728 & 0,358 & 0,282 & 0,522 & 0,742 \\
\hline \multirow{2}{*}{ Índice EQ-5D-3L } & $r$ & $-0,090$ & 0,051 & $-0,022$ & 0,061 & $-0,090$ & $-0,141$ & $-0,022$ & 0,007 \\
\hline & $\mathrm{p}$ & 0,538 & 0,727 & 0,885 & 0,679 & 0,587 & 0,391 & 0,895 & 0,969 \\
\hline \multirow{2}{*}{ SF-36: Função física } & $r$ & $-0,394$ & $-0,370$ & $-0,259$ & $-0,199$ & $-0,378$ & $-0,208$ & $-0,309$ & $-0,315$ \\
\hline & $\mathrm{p}$ & 0,005 & 0,009 & 0,076 & 0,176 & 0,019 & 0,211 & 0,063 & 0,058 \\
\hline \multirow{2}{*}{$\begin{array}{l}\text { SF-36: Desempenho } \\
\text { físico }\end{array}$} & $r$ & 0,063 & 0,224 & $-0,065$ & 0,098 & $-0,137$ & 0,035 & $-0,360$ & $-0,269$ \\
\hline & $p$ & 0,665 & 0,122 & 0,659 & 0,506 & 0,414 & 0,832 & 0,029 & 0,107 \\
\hline \multirow{2}{*}{ SF-36: Dor física } & $r$ & $-0,152$ & 0,080 & 0,020 & 0,139 & $-0,443$ & $-0,198$ & $-0,449$ & $-0,425$ \\
\hline & $\mathrm{p}$ & 0,298 & 0,585 & 0,894 & 0,347 & 0,005 & 0,234 & 0,005 & 0,009 \\
\hline \multirow{2}{*}{ SF-36: Saúde geral } & $r$ & $-0,136$ & $-0,190$ & $-0,104$ & $-0,098$ & $-0,027$ & 0,032 & 0,030 & $-0,021$ \\
\hline & $\mathrm{p}$ & 0,352 & 0,190 & 0,480 & 0,508 & 0,870 & 0,850 & 0,860 & 0,903 \\
\hline \multirow{2}{*}{ SF-36: Vitalidade } & $\mathrm{r}$ & 0,086 & $-0,175$ & $-0,021$ & $-0,054$ & 0,036 & $-0,094$ & $-0,315$ & $-0,151$ \\
\hline & $\mathrm{p}$ & 0,556 & 0,229 & 0,887 & 0,718 & 0,829 & 0,576 & 0,058 & 0,372 \\
\hline \multirow{2}{*}{ SF-36: Função social } & $r$ & $-0,066$ & $-0,199$ & $-0,172$ & $-0,069$ & 0,132 & 0,093 & 0,102 & 0,243 \\
\hline & $\mathrm{p}$ & 0,651 & 0,170 & 0,242 & 0,642 & 0,428 & 0,580 & 0,549 & 0,147 \\
\hline \multirow{2}{*}{$\begin{array}{l}\text { SF-36: Desempenho } \\
\text { emocional }\end{array}$} & $r$ & 0,173 & 0,213 & 0,246 & $-0,383$ & $-0,013$ & $-0,186$ & 0,008 & 0,174 \\
\hline & $\mathrm{p}$ & 0,234 & 0,142 & 0,093 & 0,007 & 0,938 & 0,263 & 0,960 & 0,302 \\
\hline \multirow{2}{*}{ SF-36: Saúde mental } & $r$ & 0,096 & 0,008 & 0,041 & 0,051 & 0,112 & 0,136 & $-0,089$ & 0,048 \\
\hline & $\mathrm{p}$ & 0,513 & 0,955 & 0,783 & 0,731 & 0,505 & 0,415 & 0,602 & 0,776 \\
\hline \multirow{2}{*}{ SF-36: MSF } & $r$ & $-0,295$ & $-0,095$ & $-0,145$ & $-0,067$ & 0,305 & $-0,321$ & 0,307 & 0,154 \\
\hline & $p$ & 0,039 & 0,516 & 0,324 & 0,651 & 0,063 & 0,049 & 0,065 & 0,363 \\
\hline \multirow{2}{*}{ SF-36: MSM } & $r$ & 0,192 & 0,011 & 0,065 & 0,122 & $-0,488$ & $-0,227$ & $-0,442$ & $-0,513$ \\
\hline & $p$ & 0,185 & 0,943 & 0,660 & 0,408 & 0,002 & 0,171 & 0,006 & 0,001 \\
\hline \multicolumn{3}{|c|}{$\begin{array}{l}\text { Nota: * } \mathrm{p}<0,05 \\
\text { EQ- } 5 \mathrm{D}-3 \mathrm{~L} \text { que varia entre } 0 \text { e } 1 \text {, sendo o } 1 \text { um perfeito } \\
\text { estado de saúde } \\
\text { EQ-VAS: Escala analógica visual vertical de } 0 \text { a } 100 \text {, } \\
\text { sendo } 100 \text { o melhor estado de saúde }\end{array}$} & \multicolumn{4}{|c|}{$\begin{array}{l}\text { IMC: Índice de Massa Corporal } \\
\text { Índice EQ-5D-3L: Sistema descritivo do questionário } \\
\text { MSF: Medida sumária física } \\
\text { MSM: Medida sumária mental } \\
\text { SF-36: Questionário Short Form } 36\end{array}$} & \multicolumn{2}{|c|}{$\begin{array}{l}\text { Teste: Correlação de Pearson } \\
\text { Teste: Teste t }\end{array}$} & \\
\hline
\end{tabular}


Observou-se a existência de uma correlação positiva entre o índice EQ-5D-3L e a redução das seguintes variáveis: peso (em \%, $r=0,384$; $p=0,016$ em Kg, $r=0,369 ; p=0,021), M G(r=0,336 ; p=0,036)$ e IMC $(r=0,380 ; p=0,017)$. Não foi observada correlação entre a redução de peso (\%, Kg), IMC, MG, PC e RCA e as pontuações do EQ-VAS, e as dimensões MSF e MSM do SF-36.

\section{DISCUSSÃO DOS RESULTADOS}

A maior prevalência de indivíduos do género feminino no nosso estudo pode estar relacionado com o facto da prevalência de obesidade em Portugal ser superior no género feminino (12) ou por as mulheres apresentarem uma maior preocupação com a estética, possivelmente recorrendo mais a consultas de tratamento para redução ponderal/ adiposidade $(12,13)$.

O valor médio de IMC encontrado $\left(30,6 \mathrm{Kg} / \mathrm{m}^{2}\right)$ foi inferior ao encontrado em estudos semelhantes $(14,15)$. Esta discrepância pode estar relacionado com o facto do acompanhamento nutricional ser realizado em clínica privada, pois utentes acompanhados no sistema público apresentam pior estado nutricional do que no sistema privado (16). Outro fator é a probabilidade de, no sistema público, estarem incluídos utentes a aguardar cirurgia de obesidade, que devido ao seu protocolo exigem um valor superior de IMC (17).

Os resultados encontrados quando estudada a QVRS entre indivíduos em eutrofia, pré-obesidade e obesidade são semelhantes aos verificados por outros autores, em que indivíduos com excesso ponderal são os que apresentam piores valores de QVRS $(7,9,18)$. Embora o valor médio de redução ponderal no presente estudo (4,3\%) tenha sido inferior à reportada noutros estudos com o mesmo período de intervenção, verificou-se uma redução significativa no peso, IMC, MG, PC e RCA. A redução ponderal de apenas 4,3\% numa amostra de indivíduos com IMC médio classificado em obesidade, embora borderline $\left(30,6 \mathrm{Kg} / \mathrm{m}^{2}\right)$, foi suficiente para se observarem alterações positivas na QVRS, como verificado noutros estudos (18). Com exceção da vitalidade, nenhuma dimensão mental aumentou significativamente entre o início e o término do estudo. Este resultado é semelhante ao observado por outros autores aquando da utilização do SF-36, que verificaram alterações na saúde física, mas não na saúde mental $(7,9,18)$. O mesmo se verificou na utilização do EQ-5D-3L onde existiu um aumento do EQ-VAS e do índice EQ-5D-3L, após intervenção (15). Neste estudo, as diferenças encontradas na QVRS do início e final são no entanto inferiores às encontradas por outros autores, possivelmente pelo menor tempo de intervenção nutricional (3 meses vs. 6 e 30 meses), ou porque a nossa amostra apresentava ao início melhor estado nutricional (IMC médio bordeline de obesidade vs. classe I e II de obesidade) $(14,15)$.

Quanto à influência do estado nutricional na QVRS, o presente estudo encontrou uma relação inversa entre o IMC e a QVRS $(7,9,15)$. Apesar de SØltof at al (15) observaram uma correlação negativa entre o IMC e a QVRS, em que utentes com IMC elevado apresentaram valores inferiores na maioria das dimensões da QVRS, na nossa amostra tal não foi verificado no índice EQ-5D-3L e EQ-VAS. Possivelmente pelo facto do nosso estudo ter uma amostra reduzida e um valor médio de IMC inferior. Quando analisada a relação da redução das variáveis observou-se que existia um aumento da pontuação do índice EQ-5D-3L com a redução ponderal e de MG, semelhante a outros estudos (19). O facto de a recolha de dados ser realizada em consulta conduziu à diminuição da amostra, devido à existência de desistências/ adiamentos das consultas. Ainda assim a percentagem de dropouts foi semelhante ao reportado em intervenções para redução ponderal em ambulatório (20). Os participantes eram seguidos em consulta de perda ponderal, pelo que, não se pode considerar a amostra como representativa da população com excesso ponderal, ainda assim, e apesar do número reduzido de utentes avaliados neste estudo, foram analisados diferentes parâmetros antropométricos, que poderão ter influência na perceção da QVRS. Este estudo fornece informação sobre o impacto de uma intervenção a curto prazo, na QVRS de utentes seguidos em consulta de obesidade.

\section{CONCLUSÕES}

Foram nossos objetivos, avaliar a QVRS na perda ponderal, bem como avaliar a QVRS em indivíduos com excesso ponderal/ adiposidade; e relacionar a recente perda ponderal/adiposidade com a QVRS. A redução ponderal de apenas 4,3\% numa amostra de indivíduos com IMC médio classificado em obesidade, embora borderline $\left(30,6 \mathrm{Kg} / \mathrm{m}^{2}\right)$, foi suficiente para se observarem alterações positivas na QVRS.

\section{AGRADECIMENTOS}

O presente estudo é parte integrante da tese de Mestrado em Nutrição Clínica de um dos autores AE, orientado por ACM, organizado pela Faculdade de Medicina da Universidade de Lisboa em parceria com a Escola Superior de Tecnologia da Saúde de Lisboa, Instituto Politécnico de Lisboa. Os autores agradecem aos utentes a sua participação.

\section{REFERÊNCIAS BIBLIOGRÁFICAS}

1. World Health Organization. Obesity and overweight. 2016. [Consultado em setembro de 2017]. Dsponivel em: http://www.who.int/mediacentre/factsheets/fs311/en/.

2. World Health Organization. WHOQOL: measuring quality of life. Psychol Med. 1998:551558.

3. Ferreira L. Criação da Versão Portuguesa do MOS SF-36 Parte I - Adaptação Cultural e Linguística. Acta Médica Portuguesa. 2000; 13:55-66.

4. Centers for Disease Control and Prevention. Health-Related Quality of Life (HRQOL). (2016) [Consultado em Novembro de 2017]. Dsponivel em: https://www.cdc.gov/ $\mathrm{hrqol} /$ concept.htm.

5. Healthy People 2020. Health-related quality of life and well-being. Foundation Health Measure Report.2010:1-6.

6. Ferreira L, Ferreira N, Pereira N. Contributos para a validação da versão Portuguesa do EQ-5D. Acta Médica Portuguesa. 2013;26(6):664-675.

7. Kolotkin L, Andersen R. A systematic review of reviews: exploring the relationship between obesity, weight loss and health-related quality of life. Clinical Obesity. 2017;7(5):273-289. 8. Tavares B, Nunes M, Santos M. Obesidade e qualidade de vida: Revisão da literatura. Revista Médica Minas Gerais. 2002; 20(3): 359-366.

9. Hassan K, Joshi V, Madhavan S, Amonkar M. Obesity and health-related quality of life: a cross-sectional analysis of the US population. International Journal Obesity. 2003;2:1227- 32.

10. World Health Organization. Physical status: the use and interpretation of anthropometry. World Health Organization. 1995;854:1-452.

11. Direção Geral da Saúde. Avaliação antropométrica no adulto-2013; 017/2013:1-9. 12. Lopes $C$, Torres $D$, Oliveira, $A$, Severo, $M$, Alarcão V, Guiomar $S$, et al. Inquérito Alimentar Nacional E de Atividade Física-IAN-AF.2015-2016;76-83.

13. Bjorntorp P, Bray A, Carroll K. Obesity: Preventing and Managing the Global Epidemic. WHO.2000;253.

14. Rothberg E, McEwen N, Kraftson T, Neshewat M, Fowler E, Bruant F, et al. The impact of weight loss on health-related quality-of-life: implications for cost-effectiveness analyses. Quality of Life Research. 2014;23(4):1371-1376.

15. Søltoft F, Hammer M, Kragh N. The association of body mass index and healthrelated quality of life in the general population: Data from the 2003 Health Survey of England. Quality of Life Research. 2009;18(10):1293-1299.

16. Santos H, Lima S, Souza C. Comparative study of the nutritional evolution of patients/candidates for bariatric surgery attended by the Unified Health System and the Supplemental Health Network. Ciência\&Saúde. 2014;19(5):1359-1365. 
17. Direção Geral da Saúde. Boas práticas na abordagem do doente com obesidade elegível para cirurgia bariátrica.2012;028/2012:2.

18. UI-haq Z, Mackay D, Fenwick E, Pell J. Meta-analysis of the association between body mass index and health- related quality of life among adults, asessed by the SF36. 2013;162(2).

19. Rothberg E, McEwen N, Kraftson T, Neshewat M, Fowler E, Bruant F, et al. The impact of weight loss on health-related quality-of-life: implications for cost-effectiveness analyses. Quality of Life Research. 2014;23(4):1371-1376.

20.Papadaki A, Linardakis M, Plada M, Larsen T, Baak M, et al. A multicentre weight loss study using a low calorie diet over 8 weeks: regional differences in efficacy across eight European cities.Swiss Med Wkly. 2013;143:1-9. 